\title{
Dapoxetine shown to be safe for premature ejaculation
}

Although the selective serotonin reuptake inhibitor (SSRI) dapoxetine has marketing approval in several countries for the on-demand treatment of premature ejaculation, only now have the adverse effects of the drug been comprehensively examined in a large study.

This prospective, open-label, observational, safety study enrolled otherwise healthy men with premature ejaculation (mean age 40.5 years), and assigned them to either dapoxetine $(n=6,712)$ or alternative care $(n=3,316)$. The alternative interventions included other oral drugs (such as the tricyclic antidepressant clomipramine and the SSRIs paroxetine, fluoxetine and sertraline), topical treatments or behavioural counselling.

Men in the dapoxetine group received either $30 \mathrm{mg}$ or $60 \mathrm{mg}$ doses, but could change dose depending on patient preference or if the low-dose regimen did not have the desired effect. Over

\section{Unlike previous phase III trials, no events of syncope were noted in the dapoxetine group... 77}

the course of the 12 -week study, $62 \%$ of patients in the experimental group did not have more than 10 doses of dapoxetine.

Overall, adverse events were noted in $12 \%$ of the men in the dapoxetine group and $9 \%$ in the alternative care group, with nausea, headache and vertigo being the most commonly reported effects. Very low levels of fatigue and diarrhoea were also noted. Unlike previous phase III trials, no events of syncope were noted in the dapoxetine group, and only one patient in the alternative care group (assigned to paroxetine) experienced syncope.

In the dapoxetine group, men $>65$ years were more likely to report an adverse event, whereas in the alternative care group men aged 30-39 years of age had the highest incidence of adverse events. A number of factors at baseline were associated with dapoxetine-related adverse effects, including cardiovascular disorder, metabolic disorders and alcohol consumption.

Unlike other SSRIs, dapoxetine is rapidly metabolized, which lends itself to on-demand use. Furthermore, the drug's pharmacokinetic profile probably contributes to its better safety profile compared with other SSRIs, even at a dose of $60 \mathrm{mg}$. However, in patients with contraindications (including use of antidepressant, CYP3A4 inhibitor and triptan therapies), cautious low-dosing at $30 \mathrm{mg}$ should be considered first.

Mina Razzak

Original article Mirone, V. et al. Results from a prospective observational study of men with premature ejaculation treated with dapoxetine or alternative care: the PAUSE study. Eur. Urol. doi:10.1016/j.eururo.2013.08.018 